\title{
Coherence Converting Plasmonic Hole Arrays
}

\author{
Choon How Gan • Yalong Gu • \\ Taco D. Visser • Greg Gbur
}

Received: 21 June 2011 / Accepted: 20 November 2011 / Published online: 21 January 2012

(C) Springer Science+Business Media, LLC 2012

\begin{abstract}
Simulations are presented that demonstrate that the global state of spatial coherence of an optical wavefield can be altered on transmission through an array of subwavelength-sized holes in a metal plate that supports surface plasmons. It is found that the state of coherence of the emergent field strongly depends on the separation between the holes and their scattering strength. Our findings suggest that subwavelength hole arrays on a metal film can be potentially employed as a plasmon-assisted coherence converting device, useful in modifying the directionality, spectrum, and polarization of the transmitted wave.
\end{abstract}

Keywords Plasmons • Coherence • Enhanced transmission

\section{H. Gan}

Electronics and Photonics Department, Institute of High Performance Computing Agency

for Science, Technology and Research,

1 Fusionopolis Way, Singapore, 138632, Singapore

\section{Y. Gu $\cdot$ G. Gbur $(\bowtie)$}

Department of Physics and Optical Science,

University of North Carolina at Charlotte,

9201 University City Boulevard,

Charlotte, NC 28223, USA

e-mail: gjgbur@uncc.edu

\section{T. D. Visser}

Department of Physics and Astronomy,

Free University, De Boelelaan 1081,

1081 HV Amsterdam, the Netherlands

\section{T. D. Visser}

Department of Electrical Engineering,

Delft University of Technology,

Mekelweg 4, 2628 CD Delft, the Netherlands

\section{Introduction}

Surface plasmon-mediated optical effects are now widely employed in the development of novel and improved nano-photonic devices. For instance, surface plasmons have been used to perform nanolithography [1], to develop plasmonic lasers [2], to act as a gate for light transmission [3], to improve the capabilities of nano-optical readout systems $[4,5]$, and to serve as the carrier signal in nano-optical circuits [6].

The amount of light transmitted through subwavelength-size apertures is typically low. However, it is now well-known that surface plasmons can increase the amount of light passing through an array of such apertures [7], or an appropriately structured single aperture [8]. The enhancement can be understood as arising from constructive interference between the light that is directly transmitted through the hole and light that has coupled into a plasmon; this was illustrated clearly using a plasmonic version of Young's double slit experiment [9].

Recently, through a series of investigations based on Young's double slit configuration, it has been found that surface plasmons play a central role in not only the interference and transmission of light but also the coherence properties of the transmitted light $[10,11]$. Specifically, the presence of surface plasmons in the double-slit system leads to the modulation of the spatial coherence of light that emerges from the slits, and the resulting coherence can be higher or lower than the coherence of the illuminating field.

The spatial coherence, or more specifically the spectral degree of coherence of an optical field, is a measure of the "statistical similarity" of the field at any two points and determines its interference-causing 
capability [12]. Fields that are fully coherent produce interference fringes with maximum fringe visibility, while completely incoherent fields produce no fringes. The spatial coherence influences numerous properties of the light field as it propagates, such as its directionality [13], spectrum [14], and state of polarization $[15,16]$. Because of this, partial coherence has become an important ingredient in a number of applications [17]. Therefore, any new method that would allow us to modulate the spatial coherence of a wavefield is of great interest.

The plasmon double-slit experiment described above suggests that it may be possible to alter the state of coherence of a light field as a whole, i.e., the global state of coherence, by transmitting light through an appropriate array of subwavelength-size holes in a metal plate. Such a plate will be referred to as a coherence converting plasmonic hole array. However, it is unclear how the presence of multiple holes and multiple interactions between them would affect the state of coherence of the transmitted wave. Recent research on a three-slit Young-type experiment [18] has indicated that the center slit can serve not just to decrease the effects of the plasmons propagating from one slit to the other but also to preserve and even enhance these effects; it is therefore reasonable to expect a similar effect for a two-dimensional array of holes. In a sense, our work can be regarded as complimentary to research [19] that demonstrated that the patterning of subwavelength holes can significantly affect the total transmitted power.

It is to be noted that other research has shown that plasmons can have an interesting and non-trivial effect on the statistical properties of light. For instance, it has been demonstrated $[20,21]$ that the presence of surface waves in a thermal source can produce highly directional and coherent emission. In the quantum regime, plasmon-assisted transmission of entanglement has been experimentally verified [22, 23], and decoherence effects have also been connected with plasmons [24].

In the present study, we explore the possibility of using a plasmonic hole array as a practical coherence converting device. In particular, we apply a simple cylindrical wave model for plasmons to study the change in the spatial coherence of a wavefield as it is transmitted through such an array. It is shown that it is possible to achieve a significant enhancement in the spatial coherence of the field, even approaching the limit of complete coherence. We begin by reviewing the relevant concepts from optical coherence theory and then introduce the geometry of the system and the theoretical model used to study it. Simulation results are then presented, followed by concluding remarks.

\section{Coherence in the Space-Frequency Domain}

To study the coherence of a fluctuating wavefield, we employ the space-frequency representation, in which the second-order coherence properties of the wavefield ("first order" in the terminology of quantum coherence) are characterized by the cross-spectral density function $W\left(\mathbf{r}_{1}, \mathbf{r}_{2}, \omega\right)$ defined as [25]

$W\left(\mathbf{r}_{1}, \mathbf{r}_{2}, \omega\right)=\left\langle U^{*}\left(\mathbf{r}_{1}, \omega\right) U\left(\mathbf{r}_{2}, \omega\right)\right\rangle$,

where $\mathbf{r}_{1}$ and $\mathbf{r}_{2}$ are position vectors and $U(\mathbf{r}, \omega)$ is a monochromatic realization of the field at position $\mathbf{r}$ with frequency $\omega$. Here the asterisk indicates complex conjugation, and the angular brackets $\langle\cdots\rangle$ denote averaging over the ensemble of realizations. The field $U(\mathbf{r}, \omega)$ at frequency $\omega$ satisfies the Helmholtz equation in free space,

$\left(\nabla^{2}+k^{2}\right) U(\mathbf{r})=0$,

where $k=\omega / c$ is the wavenumber.

The normalized strength of the spatial correlations between two points is characterized by the spectral degree of coherence $\mu\left(\mathbf{r}_{1}, \mathbf{r}_{2}, \omega\right)$, defined by the expression

$\mu\left(\mathbf{r}_{1}, \mathbf{r}_{2}, \omega\right)=\frac{W\left(\mathbf{r}_{1}, \mathbf{r}_{2}, \omega\right)}{\left[S\left(\mathbf{r}_{1}, \omega\right) S\left(\mathbf{r}_{2}, \omega\right)\right]^{1 / 2}}$,

where $S(\mathbf{r}, \omega)=W(\mathbf{r}, \mathbf{r}, \omega)$ is the spectral density at position $\mathbf{r}$ (the intensity of light at frequency $\omega$ ). It can be shown that $0 \leq\left|\mu\left(\mathbf{r}_{1}, \mathbf{r}_{2}, \omega\right)\right| \leq 1$, with $\left|\mu\left(\mathbf{r}_{1}, \mathbf{r}_{2}, \omega\right)\right|=1$ representing complete coherence, and $\left|\mu\left(\mathbf{r}_{1}, \mathbf{r}_{2}, \omega\right)\right|=0$ representing complete incoherence. For Young's double slit experiment with equal intensity illumination of the two slits, the modulus of the spectral degree of coherence of light emanating from the two slits is equal to the visibility [12, Sec. 4.2] of the interference fringes seen on the plane of observation, i.e.,

$\mathcal{V}(P, \omega)=\frac{S_{\max }(P, \omega)-S_{\min }(P, \omega)}{S_{\max }(P, \omega)+S_{\min }(P, \omega)}$.

Here $S_{\max }(P, \omega)$ and $S_{\min }(P, \omega)$ are the maximum and minimum intensity of the fringes in the immediate neighborhood of a point $P$ on the plane of observation. The visibility $\mathcal{V}(P, \omega)$ tends to unity if $S_{\max }(P, \omega) \gg$ $S_{\min }(P, \omega)$ and is equal to zero if no interference fringes are formed. For a multiple hole interferometer, there is no direct relationship between visibility and the 
spectral degree of coherence, as the latter quantity is typically different for each pair of holes. To assess the overall, or global, coherence of a wavefield, we must instead consider the spectral degree of coherence at relevant pairs of points in the field and look for trends in the function $\mu\left(\mathbf{r}_{1}, \mathbf{r}_{2}, \omega\right)$ as a whole. Equations 1 and 2 will be used to characterize how the spatial coherence of an incident partially coherent field is changed on transmission through the plasmonic hole array, to be discussed next.

\section{Theoretical Model}

We consider an array of subwavelength holes perforated in a thin metal film that is located at the plane $z=0$ (Fig. 1). The hole array is illuminated on the side $z<0$ by a partially coherent scalar field $U_{0}(\mathbf{r}, \omega)$, where $\mathbf{r}=(\rho, z)$; the spectral degree of coherence of this field in the plane $z=0$ is denoted as $\mu_{0}\left(\rho_{1}, \rho_{2}, \omega\right)$. It is assumed that the field $U_{0}(\mathbf{r}, \omega)$ is quasi-monochromatic and has a central wavelength $\lambda_{0}$. A fraction $\alpha$ of the incident field will be directly transmitted through the holes. Part of the field impinging on the holes may scatter and couple to surface plasmons, which can propagate along the two surfaces of the metal film. In terms of the free-space wavenumber $\left(k_{0}=2 \pi / \lambda_{0}\right)$, the in-plane wavevector of a surface plasmon mode at a metal/air interface (with relative permittivities $\epsilon_{m}$ and $\epsilon_{0}$ ) is given by $k_{\mathrm{sp}}=k_{0} \sqrt{\epsilon_{0} \epsilon_{m} /\left(\epsilon_{0}+\epsilon_{m}\right)}$, and its amplitude decays exponentially into both the metal and air [26]. When the plasmonic field propagates to a neighboring hole, it is either scattered as a plasmon or coupled back into a transmitted, freely propagating field. In general,

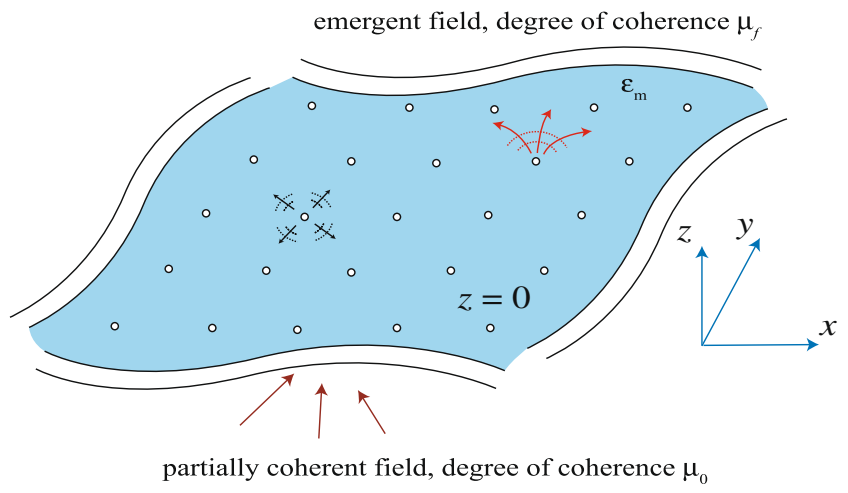

Fig. 1 Depicting the general geometry of the plasmon coherence system. Brown arrows represent the incident field, black arrows represent the in-plane plasmonic surface wave, and red arrows represent the emergent field

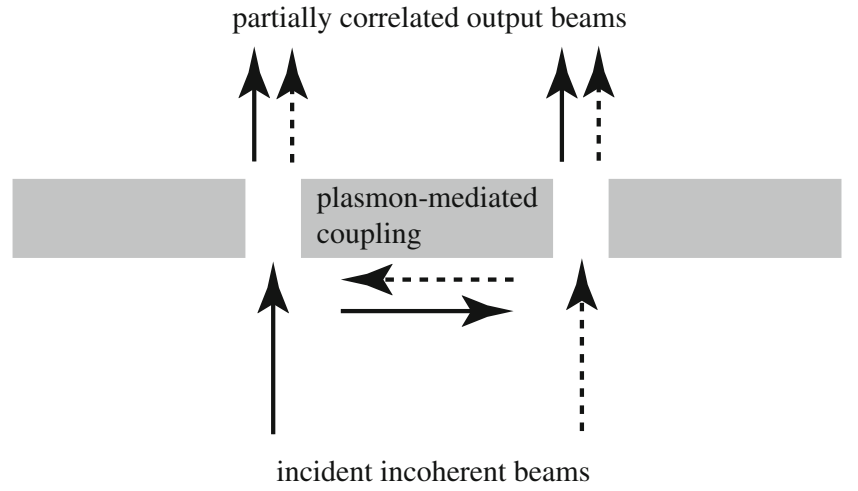

Fig. 2 Illustration of the physical mechanism behind plasmonmediated coherence enhancement for a two-slit array

the surface plasmons can be multiply scattered by the hole array before they are absorbed or recoupled into light. Interference between the field transmitted via surface plasmon coupling and the part that is directly transmitted can alter the coherence properties of light emerging from the array. We denote by $\mu_{f}\left(\mathbf{r}_{1}, \mathbf{r}_{2}, \omega\right)$ the spectral degree of coherence between any two points of the field $U_{f}(\mathbf{r}, \omega)$ emergent on the dark side of the plate.

A simple picture of this effect for two holes is depicted in Fig. 2, after [10]. Each hole is illuminated by an independent beam of light, and these two beams do not produce interference fringes. However, at the apertures, each beam is partly transmitted directly and partly coupled into a plasmonic surface wave, the latter of which travels to the other hole and is then transmitted as light. The net result is that the emission from each hole consists partly of the light from both beams, and a correlation has been introduced. Because plasmons are waves and can produce interference effects, the overall transmission and the change in coherence will depend upon the phase relation between the plasmon and directly transmitted beam and hence upon the hole separation.

The development of the theoretical model can be divided into three parts. First, the illuminating field is modeled using a coherent-mode decomposition of the field, discussed in the "Coherent-Mode Decomposition of the Illuminating Field" section. Second, the interaction of each mode of the field and the metal plate is taken into account using a multiple scattering formalism, detailed in the "Analytic Model for the Multiply Scattered Plasmonic Field" section. Finally, the coherence properties of the transmitted field are analyzed by comparing $\mu_{0}\left(\boldsymbol{\rho}_{1}, \boldsymbol{\rho}_{2}, \omega\right)$ and $\mu_{f}\left(\mathbf{r}_{1}, \mathbf{r}_{2}, \omega\right)$; this is discussed in the "Coherence of the Transmission Field" section. 
Coherent-Mode Decomposition of the Illuminating Field

The illuminating field is taken to be of Gaussian Schell model form [12, Sec. 5.3.1] in the plane $z=0$, such that

$W_{0}\left(\rho_{1}, \rho_{2}\right)=\sqrt{S_{0}\left(\boldsymbol{\rho}_{1}\right)} \sqrt{S_{0}\left(\boldsymbol{\rho}_{2}\right)} \mu_{0}\left(\boldsymbol{\rho}_{2}-\boldsymbol{\rho}_{1}\right)$.

Here $S_{0}$ represents the spectral density of the field, and $\mu_{0}$ is the spectral degree of coherence; the explicit dependence on $\omega$ is now suppressed for brevity. Schell model fields are characterized by the property that their spectral degree of coherence $\mu_{0}\left(\boldsymbol{\rho}_{1}, \boldsymbol{\rho}_{2}\right)$ at any two field points $\rho_{1}$ and $\rho_{2}$ depends only on the difference $\boldsymbol{\rho}_{2}-\boldsymbol{\rho}_{1}$; a Gaussian Schell model field is one whose spectral degree of coherence $\mu_{0}$ is of Gaussian form,

$\mu_{0}\left(\rho_{2}-\rho_{1}\right)=\exp \left(-\left|\rho_{2}-\rho_{1}\right|^{2} / 2 \delta^{2}\right)$,

where $\delta$ is the transverse correlation length.

We may rewrite Eq. 4 by expressing $\mu_{0}\left(\rho_{2}-\rho_{1}\right)$ in terms of its Fourier transform, i.e.,

$\mu_{0}\left(\boldsymbol{\rho}_{2}-\boldsymbol{\rho}_{1}\right)=\iint \tilde{\mu}_{0}(\mathbf{K}) \exp \left[i \mathbf{K} \cdot\left(\boldsymbol{\rho}_{2}-\boldsymbol{\rho}_{1}\right)\right] \mathrm{d}^{2} K$.

The cross-spectral density then takes on the form

$$
\begin{aligned}
W_{0}\left(\boldsymbol{\rho}_{1}, \boldsymbol{\rho}_{2}\right)= & \iint \tilde{\mu}_{0}(\mathbf{K})\left[\sqrt{S_{0}\left(\boldsymbol{\rho}_{1}\right)} \exp \left[i \mathbf{K} \cdot \boldsymbol{\rho}_{1}\right]\right]^{*} \\
& \times \sqrt{S_{0}\left(\boldsymbol{\rho}_{2}\right)} \exp \left[i \mathbf{K} \cdot \boldsymbol{\rho}_{2}\right] \mathrm{d}^{2} K .
\end{aligned}
$$

This expression for the cross-spectral density is of the form of an incoherent superposition of modes $\phi_{K}(\rho)$, defined as

$\phi_{K}(\boldsymbol{\rho}) \equiv \sqrt{S_{0}(\boldsymbol{\rho})} \exp [i \mathbf{K} \cdot \boldsymbol{\rho}]$.

With this definition, we may write

$W_{0}\left(\boldsymbol{\rho}_{1}, \boldsymbol{\rho}_{2}\right)=\iint \tilde{\mu}_{0}(\mathbf{K}) \phi_{K}^{*}\left(\boldsymbol{\rho}_{1}\right) \phi_{K}\left(\boldsymbol{\rho}_{2}\right) \mathrm{d}^{2} K$.

The modes are weighted by the function $\tilde{\mu}_{0}(\mathbf{K})$ which, using Eq. 5, has the form

$\tilde{\mu}_{0}(\mathbf{K})=\frac{1}{2 \pi \delta_{k}^{2}} \exp \left[-K^{2} / 2 \delta_{k}^{2}\right]$,

where we have introduced $\delta_{k} \equiv 1 / \delta$; small values of $\delta_{k}$ correspond to a high degree of spatial coherence.

We now take the spectral density of the illuminating field to be uniform across the plane $z=0$, i.e., $S_{0}(\rho)=$ $S_{0}$. Then the cross-spectral density of the field takes on the form of a weighted incoherent superposition of plane waves,

$\phi_{K}(\boldsymbol{\rho}) \equiv \sqrt{S_{0}} \exp [i \mathbf{K} \cdot \boldsymbol{\rho}]$.

Let us consider the coupling of the modes $\phi_{K}(\rho)$ from the illuminated side to the dark side of the metal plate. Regardless of the specific mechanism of coupling, provided it is linear, the modes will remain incoherent as they traverse the system; this implies that the crossspectral density of the field on the dark side of the plate may be written as

$W_{f}\left(\mathbf{r}_{1}, \mathbf{r}_{2}\right)=\iint \tilde{\mu}_{0}(\mathbf{K}) \psi_{K}^{*}\left(\mathbf{r}_{1}\right) \psi_{K}\left(\mathbf{r}_{2}\right) \mathrm{d}^{2} K$,

where $\psi_{K}(\mathbf{r})$ is the field of the $K$ th mode on the dark side, to be determined below. To evaluate the crossspectral density of the field on this side, we can independently evaluate the propagation of each individual mode and then sum them according to Eq. 12.

Equation 9 is, in essence, a coherent mode representation of the wavefield, as first introduced in [25]. In such a representation, the wavefield is decomposed into a set of mutually incoherent wavefields that are also mutually orthogonal over a given domain of integration, in this case the plane $z=0$. On traversing the metal film, the modes are no longer mutually orthogonal but remain mutually incoherent with respect to one another, as Eq. (12) holds and $\tilde{\mu}_{0}(\mathbf{K})$ does not change in the process.

\section{Analytic Model for the Multiply Scattered} Plasmonic Field

In previous research, it has been shown that relatively simple models for plasmonic interactions can provide good quantitative results. For instance, both plasmonic enhanced transmission [9] and plasmonic coherence changes [10] in Young's double slit experiment were successfully characterized by a simple plane wave model. Furthermore, in-plane scattering of surface plasmons from surface defects was well described by a simple scalar cylindrical wave model [27]. We follow this latter approach to calculate the transmission modes $\psi_{K}(\mathbf{r})$ in a manner that takes into account the effects of multiple scattering of the surface plasmons between holes. While it is recognized that a scalar model will not fully describe the effects of the surface plasmons, its simplicity offers much computing convenience and can provide insights into the fully electromagnetic problem.

In our approach, the surface plasmons propagating from an individual hole at position $\boldsymbol{\rho}_{m}$ in the metal plate to a position $\rho_{j}$ are modeled as a scalar cylindrical wave of the form

$G\left(\rho_{j}, \rho_{m}\right)=\frac{\mathrm{i}}{4} H_{0}^{(1)}\left(k_{\mathrm{sp}}\left|\rho_{j}-\rho_{m}\right|\right)$,

where $k_{\mathrm{sp}}$ is the wavenumber of the surface plasmons and $H_{0}^{(1)}$ the zeroth-order Hankel function of the first kind. 
We consider the response of a plasmonic system of $N$ holes to a plane wave with incident wavevector $\mathbf{K}$. The holes are treated as point scatterers on the surface of the metal plate; this approximation is reasonable considering the assumed subwavelength size of the holes and their multiple-wavelength separation. The field radiated by the $j$ th hole of the array will be proportional to the total field $\psi_{K}\left(\boldsymbol{\rho}_{j}\right)$ at this hole, which in turn is the sum of that part of the mode $\phi_{K}$ that is directly transmitted through the hole and the plasmonic field originating from all other holes in the system, denoted $\Psi_{K}\left(\rho_{j}\right)$. The total field may be written as

$\psi_{K}\left(\boldsymbol{\rho}_{j}\right)=\alpha \phi_{K}\left(\boldsymbol{\rho}_{j}\right)+\Psi_{K}\left(\boldsymbol{\rho}_{j}\right)$,

where $\alpha$ represents the fraction of light directly transmitted. Let $\beta$ describe the scattering strength of each of the point scatterers; one may then express $\Psi_{K}\left(\boldsymbol{\rho}_{j}\right)$ as a superposition of the field contributions originating from all the other holes in the plane of the array, i.e.,

$\Psi_{K}\left(\boldsymbol{\rho}_{j}\right)=\beta \sum_{m=1, m \neq j}^{N} G\left(\boldsymbol{\rho}_{j}, \boldsymbol{\rho}_{m}\right) \psi_{K}\left(\boldsymbol{\rho}_{m}\right)$.

Substituting from Eq. 15 for $\Psi_{K}\left(\boldsymbol{\rho}_{j}\right)$ in Eq. 14, the total field at the $j$ th scatterer is

$\psi_{K}\left(\boldsymbol{\rho}_{j}\right)=\alpha \phi_{K}\left(\boldsymbol{\rho}_{j}\right)+\beta \sum_{m=1, m \neq j}^{N} G\left(\boldsymbol{\rho}_{j}, \boldsymbol{\rho}_{m}\right) \psi_{K}\left(\boldsymbol{\rho}_{m}\right)$.

We therefore have a system of $N$ equations for the total field $\psi_{K}\left(\boldsymbol{\rho}_{j}\right)$ at each of the $N$ holes; this system of equations is often referred to as the Foldy-Lax equations [28, 29].

By writing Eq. 16 in a matrix form, the field at each of the $j$ th scatterer can be formally calculated as

$\mathbf{U}=\alpha[\mathbf{I}-\beta \mathbf{G}]^{-1} \mathbf{U}^{(\mathbf{0})}$,

where $\mathbf{U}=\left[\psi_{K}\left(\boldsymbol{\rho}_{1}\right), \psi_{K}\left(\boldsymbol{\rho}_{2}\right), \ldots, \psi_{K}\left(\boldsymbol{\rho}_{N}\right)\right]^{\mathrm{T}}, \mathbf{U}^{(\mathbf{0})}=$ $\left[\phi_{K}\left(\rho_{1}\right), \phi_{K}\left(\rho_{2}\right), \ldots, \phi_{K}\left(\rho_{N}\right)\right]^{\mathrm{T}}$, the superscript $\mathrm{T}$ refers to matrix transposition, $\mathbf{I}$ is the identity matrix, and $\mathbf{G}$ is an $N \times N$ matrix with null diagonal elements. By solving for $\mathbf{U}$, the field $\psi_{K}\left(\boldsymbol{\rho}_{j}\right)$ at each of the holes may be determined, and this field may then be substituted into Eq. 12 to evaluate $W_{f}\left(\mathbf{r}_{1}, \mathbf{r}_{2}\right)$. It is to be noted that $\alpha$ represents an overall scaling factor that plays a role in the overall light transmission but not in the normalized coherence properties of the wavefield.

We have yet to specify $\beta$, the complex parameter describing the scattering of the plasmonic field at each of the holes. The value of the modulus of $\beta$, which is a measure of the scattering strength of each hole, significantly affects the behavior of the transmitted field and must be carefully determined. To do so, we treated each hole as an electric dipole with dipole moment as given by electrostatics theory [30, Sec. 5.2] and arrived at an order of magnitude estimate of $|\beta| \sim 5$. The details leading to this estimate are given in "Appendix."

\section{Coherence of the Transmitted Field}

As the modes $\psi_{K}\left(\boldsymbol{\rho}_{j}\right)$ are associated with the twodimensional Green's function in our multiple scattering model, they collectively describe the field emerging from the holes at the plane $z=0$. The spectral degree of coherence of the transmitted field may be determined using Eqs. 12 and 2.

As noted previously, there is no definition for the global or overall degree of coherence of a wavefield. We characterize an increase in the global degree of coherence if $\left|\mu_{f}\right|>\left|\mu_{0}\right|$ for light coming from most, but not necessarily all, pairs of apertures. Because of the symmetry of the arrays considered, we only need to consider a small number of pair correlations to determine whether a majority of pairs have enhanced coherence.

\section{Simulations of Coherence Modulating Devices}

We consider the coherence properties of three different arrays of holes, illustrated in Fig. 3. A $3 \times 3$ array, a $4 \times 4$ array, and a seven-hole hexagonal array are considered. For all simulations, the free-space wavelength of light is taken to be $\lambda_{0}=600 \mathrm{~nm}$, and the metal plate is taken to be gold. The refractive index of gold at $600 \mathrm{~nm}$ is $n_{\mathrm{Au}}=0.21+\mathrm{i} 3.27$, taken from [31]. The corresponding wavelength of the surface plasmons is $\lambda_{\mathrm{sp}}=$ $2 \pi / \Re\left\{k_{\mathrm{sp}}\right\}=0.57 \mu \mathrm{m}$, and the propagation distance at which the intensity of the surface plasmons decreases to $1 / e$ is $L_{\mathrm{sp}}=\left(2 \Im\left\{k_{\mathrm{sp}}\right\}\right)^{-1} \approx 7 \mu \mathrm{m}$. The distance between neighboring holes in the array is kept shorter than $L_{\mathrm{sp}}$

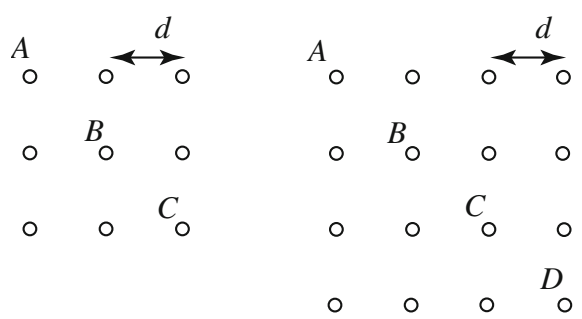

(a) (b)

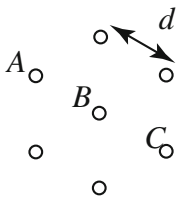

(c)
Fig. 3 The three hole geometries under consideration: a $3 \times 3$ square hole array, b $4 \times 4$ hole array, and $\mathbf{c}$ a seven-hole hexagonal array 
in the simulations so that the surface plasmon effects remain significant. The value of $|\beta|$ is taken to be 5 , unless otherwise stated. As a simple check on the model, we verified that $\mu_{f} \longrightarrow \mu_{0}$ as $|\beta| \longrightarrow 0$ in our simulations, i.e., the modulation of the spatial coherence depends on the presence of surface plasmons in the system.

We consider the behavior of the $3 \times 3$ hole array first. Figure $4 \mathrm{a}$ shows the absolute value of the spectral degree of coherence $\mu_{f}$ between holes $A$ and $C$ as a function of the hole separation $d$; the degree of coherence of the illuminating field is shown as a dashed line for comparison. It can be seen that the coherence of the output field between the two holes is in general greater than the coherence of the input field. Most notably, there exist very significant increases of coherence for $d \approx 1.1 \mu \mathrm{m}, d \approx 1.4 \mu \mathrm{m}$, and $d \approx 1.9 \mu \mathrm{m}$. Such increases can only be said to be global increases, however, if they occur for most pairs of holes in the system. In Fig. 4b, the degree of coherence of hole pairs $(A, B)$ and $(A, C)$ are compared. It can be seen that the peaks of the greatest enhancements coincide; due to the symmetry of the hole system, this implies that the coherence between most pairs of holes dramatically increases at the same separations $d$.

It is of interest to ask whether the location of these resonant peaks depend on the value of $\mu_{0}$. In Fig. 5, the coherence between holes $A$ and $C$ as a function of $d$ is shown for several values of the incident degree of

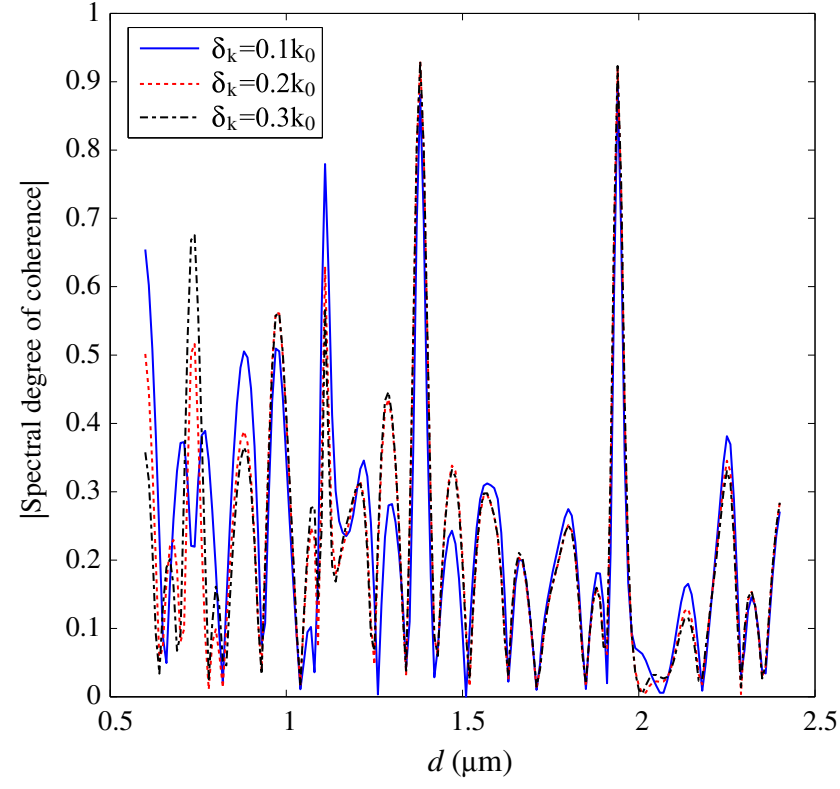

Fig. 5 Illustrating the dependence of the degree of coherence of the $3 \times 3$ square hole array on the degree of coherence of the illuminating field

coherence. It can be seen that, though the degree of enhancement varies, the resonances occur for essentially the same values of $d$.

For a $4 \times 4$ hole array, the relationship between coherence enhancement and $d$ becomes more complicated,

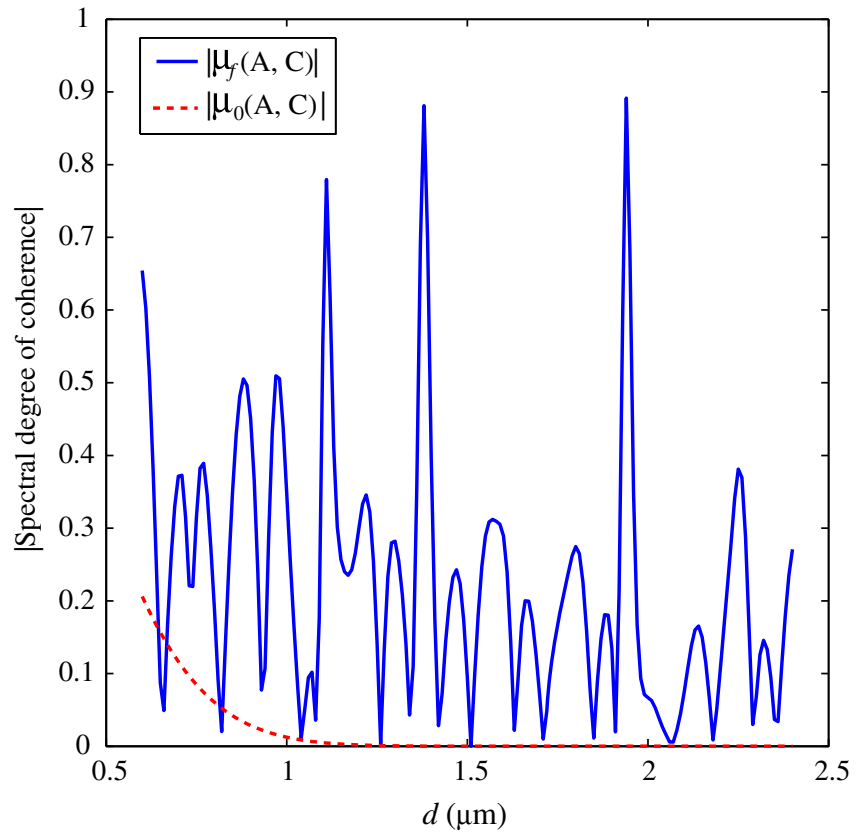

(a)

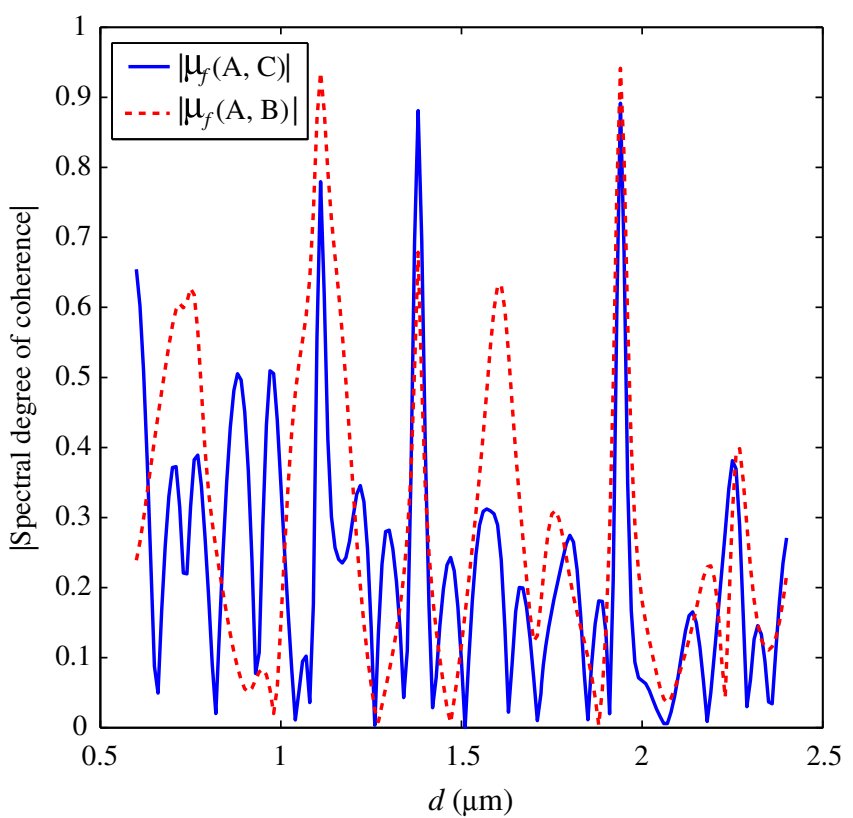

(b)

Fig. 4 The absolute value of a the spectral degree of coherence between points $A$ and $C$ in the $3 \times 3$ hole array, as a function of hole separation $d$, and $\mathbf{b}$ the spectral degree of coherence between multiple points in the array. Here $\delta_{k}=0.1 k_{0}$ 


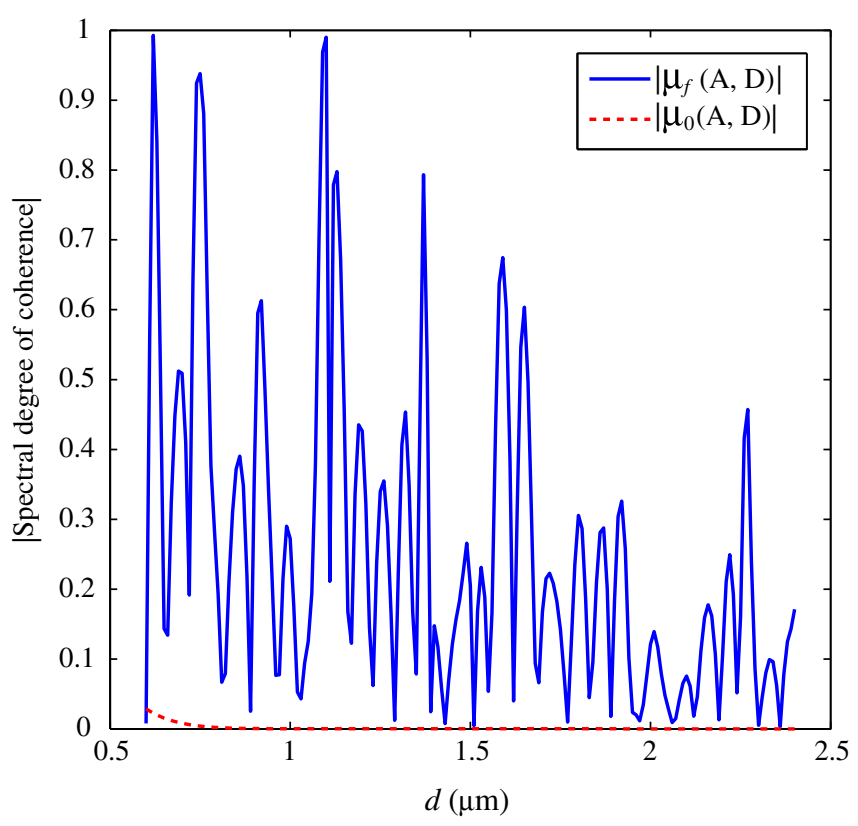

(a)

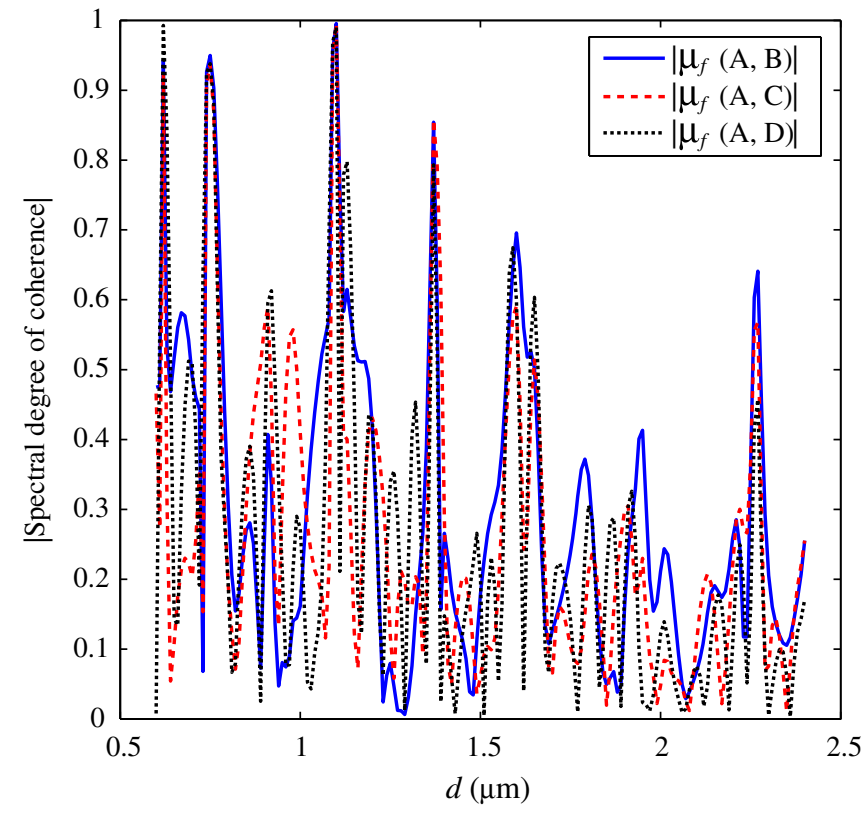

(b)

Fig. 6 The absolute value of a the spectral degree of coherence between points $A$ and $D$ in the $4 \times 4$ hole array, as a function of hole separation $d$, and $\mathbf{b}$ the spectral degree of coherence between multiple points in the array. Here $\delta_{k}=0.1 k_{0}$

but the same qualitative behaviors can be seen. In Fig. 6a, the degree of coherence between holes $A$ and $D$ are shown, and the degree of coherence of the incident field is shown for comparison. In Fig. 6b, it can be seen that a number of coherence increases are global and occur for multiple pairs of holes, notably those at $d \approx 0.75 \mu \mathrm{m}, d \approx 1.1 \mu \mathrm{m}, d \approx 1.4 \mu \mathrm{m}, d \approx 1.7 \mu \mathrm{m}$, and $d \approx 2.3 \mu \mathrm{m}$.

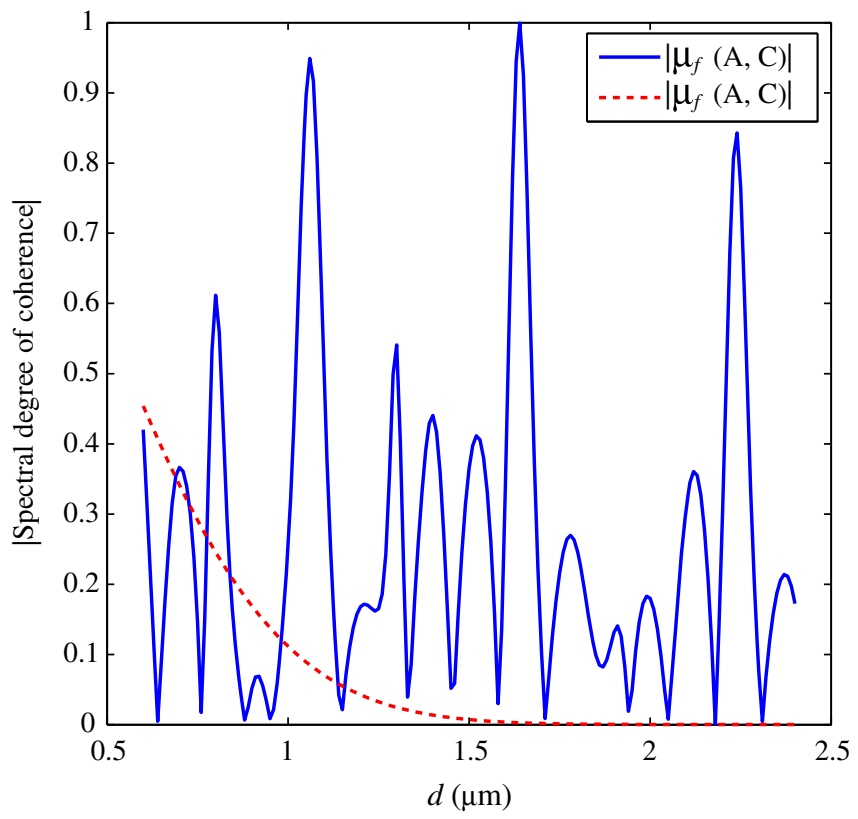

(a)

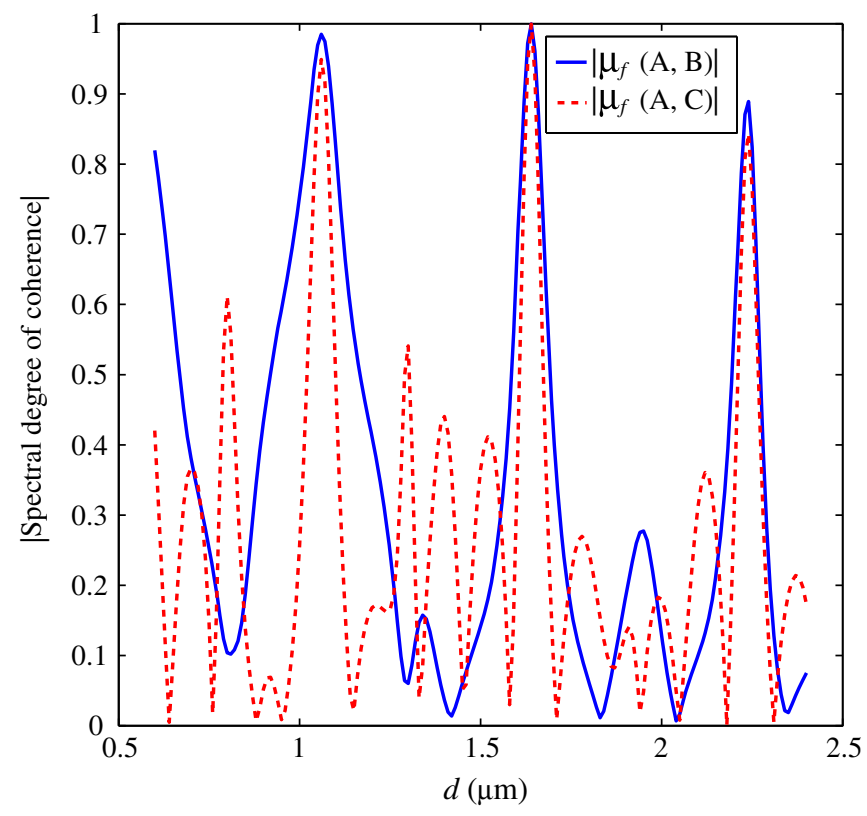

(b)

Fig. 7 The absolute value of a the spectral degree of coherence between points $A$ and $C$ in the seven-hole hexagonal array, as a function of hole separation $d$, and $\mathbf{b}$ the spectral degree of coherence between multiple points in the array. Here $\delta_{k}=0.1 k_{0}$ 
Similar results hold for different geometries of holes. The results for a seven-hole hexagonal array are presented in Fig. 7. Three peaks stand out as global increases of coherence, namely $d \approx 1.1 \mu \mathrm{m}, d \approx 1.7 \mu \mathrm{m}$, and $d \approx 2.25 \mu \mathrm{m}$.

The effectiveness of the plasmonic coherence enhancement depends crucially upon the strength of plasmonic coupling $\beta$. Figure 8 repeats the calculation of Fig. 7a but with $\beta=1$. As can be seen, the enhancement is much less significant.

In all of the previous simulations, a global enhancement of coherence can be seen, but there is no clear example of a global suppression of coherence. To ascertain whether such a suppression can occur, simulations were performed for a hexagonal array with a higher degree of coherence of the incident field, $\delta_{k}=$ $0.05 k_{0}$; the results are shown in Fig. 9. Though the points of enhancement are clearly overlapping, there are no values of $d$ for which $\mu_{f}(A, B)$ and $\mu_{f}(A, C)$ are simultaneously suppressed. It seems that a global suppression of coherence does not occur, at least in the simple hole geometries considered here.

Looking at Fig. 9 again, it can be seen that the enhancement peaks occur at regularly spaced intervals in $d$. This distance is approximately the plasmon wavelength, $\lambda_{\mathrm{sp}}=0.57 \mu \mathrm{m}$, which agrees with the earlier argument that enhancement is due to a constructive interference between the plasmonic wave and the directly transmitted wave. For the $3 \times 3$ and $4 \times 4$ hole arrays,

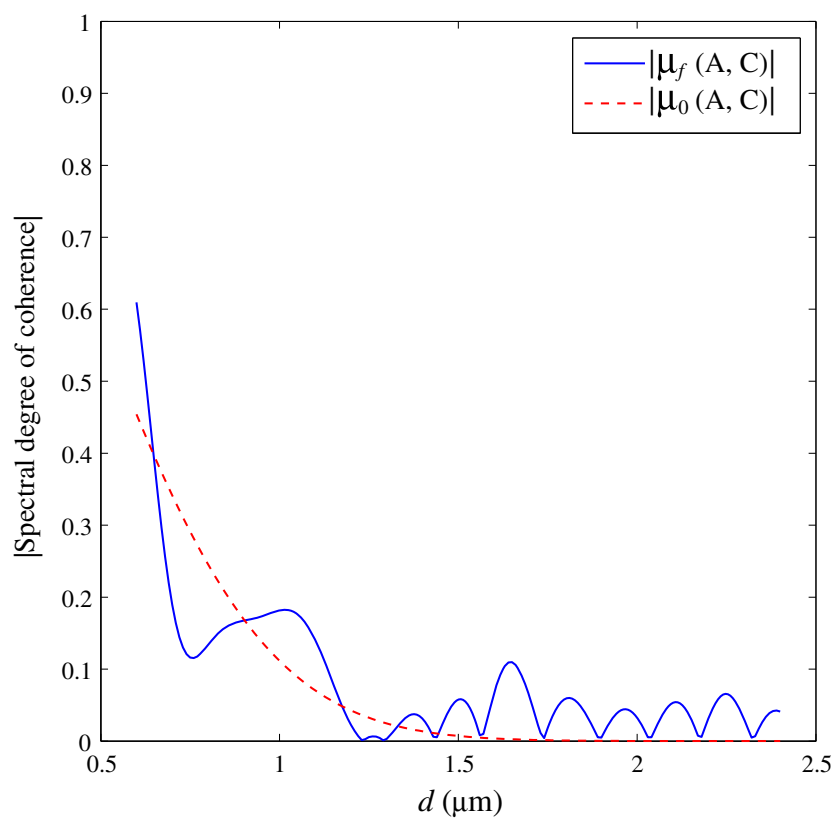

Fig. 8 The absolute value of the spectral degree of coherence between points $A$ and $C$ in the seven-hole hexagonal array, as a function of hole separation $d$, with $\delta_{k}=0.1 k_{0}$ and $\beta=1$

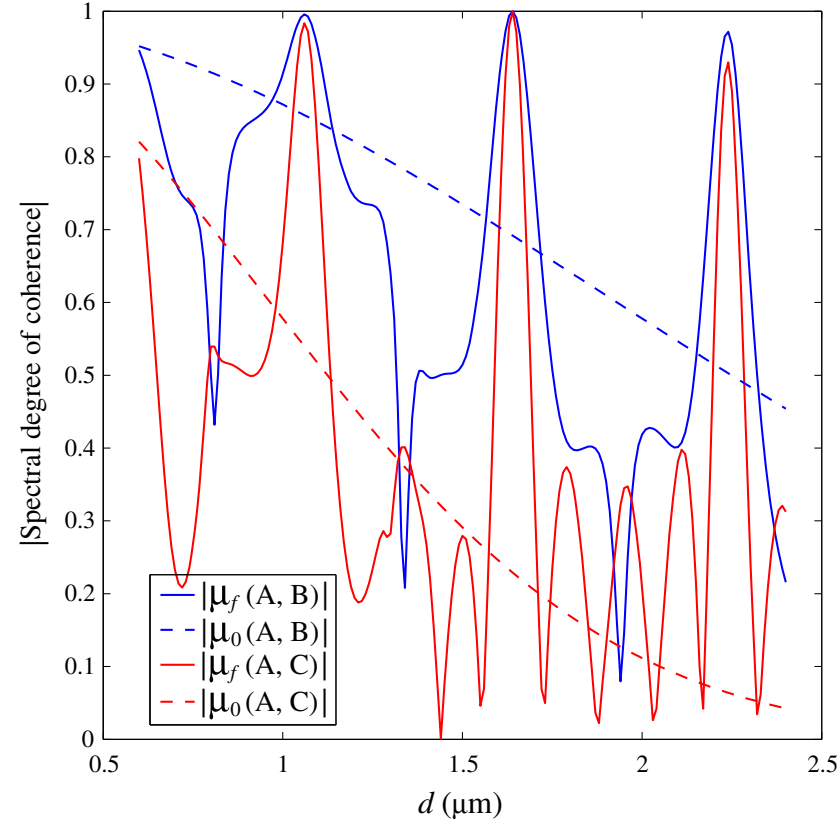

Fig. 9 The absolute value of the spectral degree of coherence between points $A$ and $C$ in the seven-hole hexagonal array, as a function of hole separation $d$, with $\delta_{k}=0.05 k_{0}$. The dashed lines indicate the coherence of the illuminating field at the holes

however, there is no clear relationship between the wavelength and the resonance positions. This is perhaps understandable, as even a simple square array has many different paths by which a plasmon can travel between holes and therefore many different length scales. For instance, a plasmon can travel a direct diagonal distance between holes, in which case it has traveled $\sqrt{2} d$. For the hexagonal array, most paths between holes are either distance $d$ or $2 d$.

\section{Conclusions}

We have demonstrated that the coherence properties of a planar Gaussian Schell model field can be modulated with a metallic film perforated with subwavelength holes. When the effects of the surface plasmons are significant, the degree of coherence of the field emerging from the array depends on the separation between the holes and their scattering strength. For larger hole arrays, the coherence peaks become much narrower and more erratic, chiefly due to the variety of path lengths that the plasmons can travel. It seems likely that breaking the symmetry of the array can help to "smooth out" the sharp resonances.

Our simulations show that the emergent field can indeed have a degree of coherence that is quite different from that of the Gaussian Schell model field. These 
results indicate that subwavelength hole arrays on a metal film can be potentially employed in nano-optical systems as a coherence converting device.

Acknowledgments Choon How Gan was supported by the Department of Energy under grant no. DE-FG02-06ER46329. Yalong Gu was supported by Air Force Office of Scientific Research under grant no. FA9550-08-1-0063. Taco Visser acknowledges support from The Netherlands Foundation for Fundamental Research of Matter (FOM).

\section{Appendix: Derivation of the Estimate of $|\beta|$}

Here we provide details of the assumptions and approximations used to arrive at the order of magnitude estimate of the scattering strength of $|\beta| \sim 5$. The derivation adheres closely to the electrostatics approximation employed by Bohren and Huffman in the problem of scattering of light by a sphere that is small compared with the wavelength [30, Sec 5.2]. A spherical cavity in a metal background medium is used as an approximation for a hole in a metal plate.

We begin by considering the electric field radiated by a monochromatic electric dipole into the far field [32, Sec 9.2], with spatial dependence

$\mathbf{E}_{s}=\frac{k^{2}}{4 \pi \epsilon_{m}}(\hat{\mathbf{r}} \times \mathbf{p}) \times \hat{\mathbf{r}} \frac{\exp (\mathrm{i} k r)}{r}$

with $k$ is the wavenumber, $\epsilon_{m}$ is the dielectric constant of the medium in which the wave propagates, $\mathbf{p}$ is the electric dipole moment, and $\hat{\mathbf{r}}$ is a unit vector in the direction of $\mathbf{r}$, which is the position vector of the point of observation as measured from the dipole $(r=|\mathbf{r}|)$. We may treat the scattering of the sphere by replacing it with an electric dipole with dipole moment $\mathbf{p}=$ $\epsilon_{m} \alpha E_{0} \exp (i \omega t) \hat{\mathbf{p}}$, where $E_{0}$ is the amplitude of the incident field illuminating the dipole, polarized along the unit vector $\hat{\mathbf{p}}$, and the polarizability of the sphere $\alpha$ is given by [32, $\mathrm{Sec} 4.4]$

$\alpha=4 \pi a^{3} \frac{\epsilon_{s}-\epsilon_{m}}{\epsilon_{s}+2 \epsilon_{m}}$

with $\epsilon_{s}$ and $a$ the dielectric constant and radius of the sphere, respectively. Substituting for p into Eq. 18 and rearranging, we have

$\mathbf{E}_{s}=E_{0} \mathbf{X} \frac{\exp (\mathrm{i} k r)}{\mathrm{i} k r}$,

where

$\mathbf{X}=\frac{\mathrm{i} k^{3}}{4 \pi} \alpha(\hat{\mathbf{r}} \times \hat{\mathbf{p}}) \times \hat{\mathbf{r}}$

is the vector scattering amplitude, a dimensionless quantity. In our model, we treat the plasmon scattering parameter $\beta$ as a scalar analog to the vector scattering amplitude $\mathbf{X}$. To estimate $|\beta|$, we thus attempt to derive an estimate for $|\mathbf{X}|$, i.e., $|\beta| \sim|\mathbf{X}|$. We note that $\alpha$ as given in Eq. 19 is the polarizability for a sphere of $\epsilon_{s}$ embedded in a medium of $\epsilon_{m}$. we are interested in the case of an air-filled spherical cavity $\left(\epsilon_{s}=\epsilon_{0}=1\right)$ for which the cavity polarizability takes on the form

$\alpha=4 \pi a^{3} \frac{1-\epsilon_{m} / \epsilon_{0}}{1+2 \epsilon_{m} / \epsilon_{0}}$.

Substituting this expression for $\alpha$ in Eq. 21, it is found that

$$
\begin{aligned}
|\mathbf{X}| & =\frac{k^{3}}{4 \pi}|\alpha| \\
& =(2 \pi)^{3} \frac{a^{3}}{\lambda^{3}}\left|\frac{1-\epsilon_{m} / \epsilon_{0}}{1+2 \epsilon_{m} / \epsilon_{0}}\right| .
\end{aligned}
$$

For metals that are conducive to the generation of surface plasmons, $\left|\operatorname{Re}\left(\epsilon_{m}\right)\right| \gg\left|\operatorname{Im}\left(\epsilon_{m}\right)\right|$, and $\left|\operatorname{Re}\left(\epsilon_{m}\right)\right| \gg$ 1 , typically. Taking this into account, then for a subwavelength sphere with radius $a=\lambda / 3$, it is obtained that

$$
|\beta| \sim|\mathbf{X}| \sim 5 .
$$

It is to be noted that while the dipole approximation is shown to be valid by Bohren and Huffman for a small sphere in a three-dimensional homogeneous medium, we have applied the formalism to obtain an order of magnitude estimate for $|\beta|$, which describes the scattering by a cylindrical hole in a two-dimensional plate. Provided the depth of the hole is not significantly larger than its diameter (i.e., the hole is as wide as it is tall), we expect that the scattering results for a sphere will not differ significantly from that of a cylinder, especially considering the assumed subwavelength size of the scatterer. It should be noted that the depth of the hole is not specified in the model used in this paper, but for plasmonic transmission experiments is often taken to be comparable to the width (see, for instance, [9]). The result $|\beta| \sim 5$ lies within the range of values found by others for similar plasmonic systems; for instance, [27] finds a value of $\beta \approx 3$, while [33] finds values as large as $\beta \approx 60$.

\section{References}

1. Liu Z-W, Wei Q-H, Zhang X (2005) Surface plasmon interference nanolithography. Nano Lett 5:957-961

2. Bergman DJ, Stockman MI (2003) Surface plasmon amplification by stimulated emission of radiation: quantum 
generation of coherence surface plasmons in nanosystems. Phys Rev Lett 90:027402

3. Janke C, Rivas JG, Bolivar PH, Kurz H (2005) All-optical switching of the transmission of electromagnetic radiation through subwavelength apertures. Opt Lett 30:2357-2359

4. Gan CH, Gbur G (2006) Strategies for employing surface plasmons in near-field optical readout systems. Opt Express 14:2385-2397

5. Gan CH, Gbur G (2007) Strategies for employing surface plasmons in a near field transmission optical readout system. Appl Phys Lett 91:131109

6. Takahara J, Kobayashi T (2004) Low-dimensional optical waves and nano-optical circuits. Opt Photon News 15: 54-59

7. Ebbesen TW, Lezec HJ, Ghaemi HF, Thio T, Wolff PA (1998) Extraordinary optical transmission through subwavelength hole arrays. Nature 391:667-669

8. Thio T, Pellerin KM, Linke RA, Lezec HJ, Ebbesen TW (2001) Enhanced light transmission through a single subwavelength aperture. Opt Lett 26:1972-1974

9. Schouten HF, Kuzmin N, Dubois G, Visser TD, Gbur G, Alkemade PF, Blok H, 't Hooft GW, Lenstra D, Eliel ER (2005) Plasmon-assisted two-slit transmission: Young's experiment revisited. Phys Rev Lett 94:053901

10. Gan CH, Gbur G, Visser TD (2007) Surface plasmons modulate the spatial coherence of light in Young's interference experiment. Phys Rev Lett 98:043908

11. Kuzmin N, 't Hooft GW, Eliel ER, Gbur G, Schouten HF, Visser TD (2007) Enhancement of spatial coherence by surface plasmons. Opt Lett 32:445-447

12. Wolf E (2007) Introduction to the theory of coherence and polarization of light. Cambridge University Press, Cambridge

13. Wolf E (1978) Coherence and radiometry. J Opt Soc Am 68:6-17

14. Wolf E, James DFV (1996) Correlation-induced spectral changes. Rep Prog Phys 59:771-818

15. James DFV (1994) Change of polarization of light beams on propagation in free space. J Opt Soc Am A 11:1641-1643

16. Wolf E (2003) Correlation-induced changes in the degree of polarization, the degree of coherence and the spectrum of random electromagnetic beams on propagation. Opt Lett 28:1078-1080

17. Gbur G, Visser TD (2010) The structure of partially coherent fields. In: Wolf E (ed) Progress in optics, vol 55. Elsevier, Amsterdam, pp 285-341
18. Gan CH, Gbur G (2008) Spatial coherence conversion with surface plasmons using a three-slit interferometer. Plasmonics 3:111-117

19. van der Molen KL, Klein Koerkamp KJ, Enoch S, Segerink FB, van Hulst NF, Kuipers L (2005) Role of shape and localized resonances in extraordinary transmission through periodic arrays of subwavelength holes: experiment and theory. Phys Rev B 72:045421

20. Greffet J-J, Carminati R, Joulain K, Mulet J-P, Mainguy S, Chen Y (2002) Coherent emission of light by thermal sources. Nature 416:61-64

21. Laroche M, Arnold C, Marquier F, Carminati R, Greffet J-J, Collin S, Bardou N, Pelouard J-L (2005) Highly directional radiation generated by a tungsten thermal source. Opt Lett 30:2623-2625

22. Altewischer E, van Exter MP, Woerdman JP (2002) Plasmonassisted transmission of entangled photons. Nature 418:304 306

23. Fasel S, Robin F, Moreno E, Erni D, Gisin N, Zbinden H (2005) Energy-time entanglement preservation in plasmonassisted light transmission. Phys Rev Lett 94:x110501

24. Altewischer E, Oei YC, van Exter MP, Woerdman JP (2005) Quantum decoherence versus classical depolarization in nanohole arrays. Phys Rev A 72:013817

25. Wolf E (1982) New theory of partial coherence in the spacefrequency domain. Part I: spectra and cross spectra of steadystate sources. J Opt Soc Am 72:343-351

26. Raether H (1988) Surface plasmons on smooth and rough surfaces and on gratings. Springer, Berlin

27. Bozhevolnyi SI, Coello V (1998) Elastic scattering of surface plasmon polaritons: modeling and experiment. Phys Rev B 58:10899

28. Foldy LL (1945) The multiple scattering of waves. Phys Rev 67:107-119

29. Lax M (1952) Multiple scattering of waves. II. The effective field in dense systems. Phys Rev 85:621-629

30. Bohren CG, Huffman DR (1983) Absorption and scattering of light by small particles. Wiley, New York

31. Johnson PB, Christy RW (1972) Optical constants of the noble metals. Phys Rev B 6:4370-4379

32. Jackson JD (1975) Classical electrodynamics, 2nd edn. Wiley, New York

33. Bozhevolnyi SI, Volkov VS (2001) Multiple-scattering dipole approach to modeling of surface plasmon polariton band gap structures. Opt Commun 198:241-245 